\title{
Magnetic Rayleigh-Taylor Instability in an Experiment Simulating a Solar Loop
}

\author{
Yang Zhang (iD), Pakorn Wongwaitayakornkul(iD, and Paul M. Bellan (iD \\ Applied Physics, California Institute of Technology, Pasadena, CA 91125, USA; yangzhan@caltech.edu \\ Received 2019 November 22; revised 2020 January 10; accepted 2020 January 14; published 2020 January 29
}

\begin{abstract}
A hoop force driven magnetic Rayleigh-Taylor instability (MRTI) is observed in a laboratory experiment that simulates a solar coronal loop. Increase of the axial wavelength $\lambda$ is observed when the axial magnetic field increases. This scaling is consistent with the theoretical MRTI growth rate $\gamma^{2}=g k-2\left(\boldsymbol{k} \cdot \boldsymbol{B}_{0}\right)^{2} / \mu_{0} \rho$, which implies that if $\boldsymbol{k}$ is parallel to $\boldsymbol{B}_{0}$ (i.e., undular mode), the fastest-growing mode has $\lambda=2 \pi / k=8 \pi B_{0}^{2} / \mu_{0} \rho g$.

Unified Astronomy Thesaurus concepts: Magnetohydrodynamics (1964); Laboratory astrophysics (2004); Solar activity (1475); Solar corona (1483); Solar prominences (1519); Solar filaments (1495); Quiescent solar prominence (1321); Solar coronal plumes (2039); Solar coronal mass ejections (310); Space plasmas (1544)
\end{abstract}

\section{Introduction}

The Rayleigh-Taylor instability (RTI) is an important instability in many astrophysical and laboratory systems, such as supernova explosions (Hachisu et al. 1992; Hester et al. 1996; Porth et al. 2014), solar prominences (Berger et al. 2010; Ryutova et al. 2010; Terradas et al. 2015; Hillier 2018), and inertial confinement fusion (Takabe et al. 1985; Betti et al. 1998). RTI occurs when a heavy fluid is initially on top of a light fluid. If the low-density fluid is vacuum, the interface is planar, and there is no magnetic field, the growth rate of this one-dimensional instability is

$$
\gamma=\sqrt{g k}
$$

where $g$ is the gravitational acceleration and $k$ is the spatial wavenumber. The instability grows as $\exp (\gamma t)$ with a ripple structure initially followed by later development of plumes and finger-like structures. For a plasma supported above vacuum by a magnetic field parallel to the planar interface, assuming that there is no magnetic field shear near the interface and the conducting wall is far away from the magnetic RayleighTaylor instability (MRTI) location (Kruskal \& Schwarzschild 1954; Goedbloed et al. 2019), the growth rate reduces to

$$
\gamma^{2}=g k-\frac{2\left(\boldsymbol{k} \cdot \boldsymbol{B}_{0}\right)^{2}}{\mu_{0} \rho}
$$

where $\boldsymbol{B}_{0}$ is the unperturbed magnetic field. For a perturbation with $\boldsymbol{k} \perp \boldsymbol{B}_{0}$, also known as an interchange mode, the growth rate is the same as the RTI without a magnetic field. However, for a perturbation with $\boldsymbol{k} \| \boldsymbol{B}_{0}$, known as an undular mode, the growth rate is

$$
\gamma^{2}=g k-\frac{2\left(k B_{0}\right)^{2}}{\mu_{0} \rho} .
$$

The undular mode has a critical wavelength

$$
\lambda_{\mathrm{c}}=\frac{4 \pi B_{0}^{2}}{\mu_{0} \rho g}
$$

at which $\gamma=0$. If $\lambda<\lambda_{c}, \gamma^{2}<0$, which implies that only perturbations with wavelength $\lambda>\lambda_{\mathrm{c}}$ can grow. Equation (3) also shows that there is a fastest-growing wavelength, which is given by

$$
\lambda_{\mathrm{m}}=2 \lambda_{\mathrm{c}}=\frac{8 \pi B_{0}^{2}}{\mu_{0} \rho g}
$$

and which corresponds to a maximum growth rate

$$
\gamma_{\mathrm{m}}=\sqrt{\frac{g k}{2}} .
$$

Zhai \& Bellan (2016) derived the MHD theory of the MRTI on the surface of a magnetically confined cylindrical plasma flux rope. The Rayleigh-Taylor instability is found to couple to the classic current-driven instability, resulting in a new type of hybrid instability.

The MRTI is thought to be the mechanism for the formation and dynamics of plumes in solar prominences. Berger et al. (2010) observed the upflows from plumes caused by the MRTI. Ryutova et al. (2010) described how the theoretically predicted growth rates and behaviors for the MRTI matched observations of quiescent prominence plumes. Hillier et al. (2012) verified the MRTI mechanism for upflows from simulations. Keppens et al. (2015) found the indications of secondary KelvinHelmholtz instabilities due to shear flows at the bubbles. However, the dependence on magnetic field strengths and how this dependence affects observed differences is still not determined. An example of such differences is that some prominences produce many small plumes while others produce only large plumes (Berger et al. 2010).

Previous experiments (Lebedev et al. 1998; Sinars et al. 2010; de Grouchy et al. 2018) investigated the MRTI using Zpinch implosions, but these experiments focused mainly on the growth rate and X-ray production and did not vary the magnetic field to test for a relation between magnetic field and the MRTI wavelength.

In the Caltech single-loop experiment, a magnetized plasma loop is generated to simulate solar flux ropes. Mechanisms for flux rope expansion (Hansen \& Bellan 2001; Ha \& Bellan 2016), magnetically driven flows (Stenson \& Bellan 2012), apex dips (Wongwaitayakornkul et al. 2017), and coronal mass ejection cavity formation (Haw et al. 2018) have been studied. We show here the existence of a hoop force driven MRTI in this experiment. Detailed measurements indicate a scaling where the observed axial wavelength $\lambda$ 


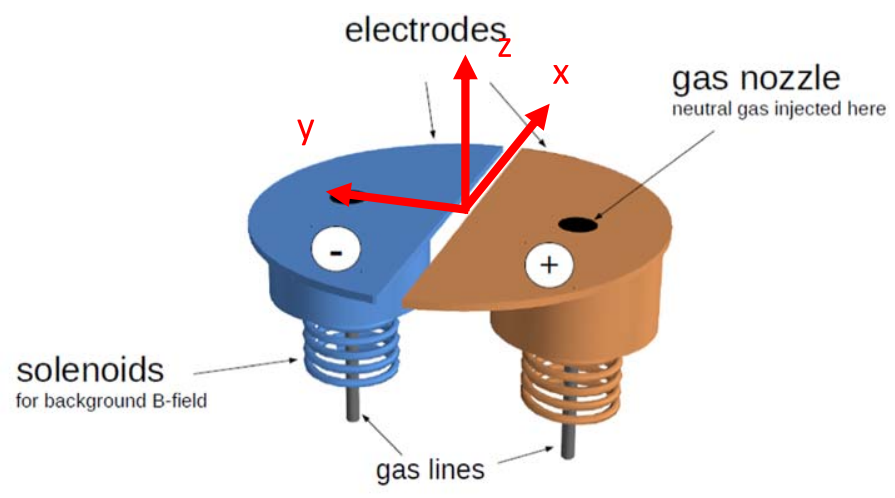

(1)

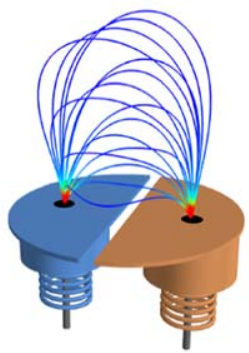

Generate background B-field
(2)

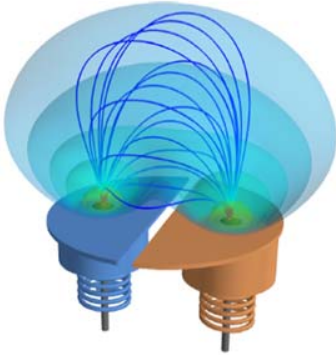

Inject neutral gas
(3)

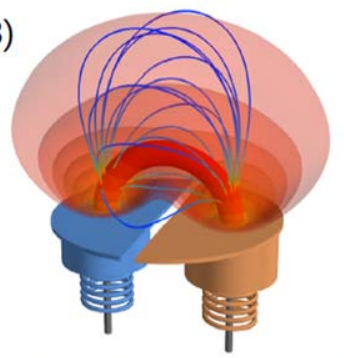

Switch 3-6 kV across electrodes, ionize neutral gas

\section{$\mathrm{t}=-5.6 \mathrm{~ms}$}

$\mathrm{t}=-4.5 \mathrm{~ms}$

$\mathrm{t}=0 \mathrm{~ms}$

Figure 1. Diagram of the single-loop apparatus showing the primary steps to generate a flux rope: (1) generate arched bias background magnetic field, (2) puff in neutral gas, and (3) switch capacitor bank across electrodes (adapted from Haw 2018 with permission).

increases with axial magnetic field, and this scaling is shown to be consistent with the MRTI.

\section{Experimental Setup}

The experimental plasma is generated in a $1.5 \mathrm{~m}$ long, $1 \mathrm{~m}$ diameter stainless steel chamber having $\sim 10^{-7}$ Torr base pressure. The chamber is considerably larger than the plasma and simulates a half-infinite space. The firing sequence is shown in Figure 1. An arched 0.01-0.3 T vacuum magnetic field called the bias field is produced by solenoids located behind the electrodes on a slow (ms) timescale so as to penetrate the electrodes. Gas is then injected into the electrode region by fast gas valves. High voltage $(3-6 \mathrm{kV})$ is applied across the electrodes on a fast $(\mu \mathrm{s})$ timescale, breaking down the gas and creating plasma. The plasma is almost fully ionized, and has density $n \sim 10^{20}-10^{21} \mathrm{~m}^{-3}$ and temperature $\sim 2-10 \mathrm{eV}$. The plasma duration is $\sim 10 \mu \mathrm{s}$. The distance between the centers of the two electrodes is $8 \mathrm{~cm}$ (Ha 2016; Haw 2018). Figure 1 defines a Cartesian coordinate system with origin at the midpoint between electrodes, $z$ along the vertical direction, and $y$ along the line between the two electrode centers. The capacitor powering the solenoids is charged to a voltage $V_{b}$, so the bias magnetic field provided by the solenoids can be expressed as $B=\alpha V_{b}$, where $\alpha$ is a constant. Vacuum measurements of the bias field were made for $V_{b}=50 \mathrm{~V}$ at $x=0 \mathrm{~cm}, z=3.81 \mathrm{~cm}$ for two different values of $y$. These give $\boldsymbol{B}=(0.00,0.02,0.08) \mathrm{T}$ at $y=$ $5.08 \mathrm{~cm}$ and $\boldsymbol{B}=(0.00,0.04,0.03) \mathrm{T}$ at $y=7.62 \mathrm{~cm}$.

\section{Experimental Results}

The image in Figure 2 shows a typical observed MRTI. The MRTI occurs in a positive $y$ region (upper region) with $y=5-8 \mathrm{~cm}, z=1-4 \mathrm{~cm}$ as determined from the images. The growth rate $\gamma_{\text {observed }}$ is determined by measuring the MRTI amplitude at a sequence of times. The effective gravitational acceleration $g_{\text {effective }}$ is determined from the $y$-direction motion of the loop at the location where the MRTI takes place. The wavelength $\lambda$ is obtained from the image when the MRTI first appears and ranges from 0.5 to $2.5 \mathrm{~cm}$. We assume the observed MRTI is the fastest-growing mode and compare the calculated growth rate $\gamma_{\text {calculated }}=\gamma_{\max }=\sqrt{\pi g_{\text {observed }} / \lambda}$ to the observed growth rate. The table in Figure 2 presents this comparison and shows that the observed growth rate is in good agreement with the calculated growth rate; this confirms that the observed structure is the MRTI.

Another question concerns the mechanism providing the effective gravity. We now show that this mechanism is provided by the hoop force acceleration. A circular, currentcarrying hoop (Shafranov 1966) with major radius $R$, minor radius $a$, internal inductance per unit length $l_{\mathrm{i}}$, and current $I$ experiences an outward radial force per unit length

$$
f_{\text {hoop }}=\frac{\mu_{0} I^{2}}{4 \pi R}\left[\ln \left(\frac{R}{a}\right)+1.08+\frac{l_{\mathrm{i}}}{2}\right] .
$$

Assuming the current is uniformly distributed, $l_{\mathrm{i}}=0.5$, so

$$
f_{\text {hoop }}=\frac{\mu_{0} I^{2}}{4 \pi R}\left[\ln \left(\frac{R}{a}\right)+1.33\right] \text {, }
$$




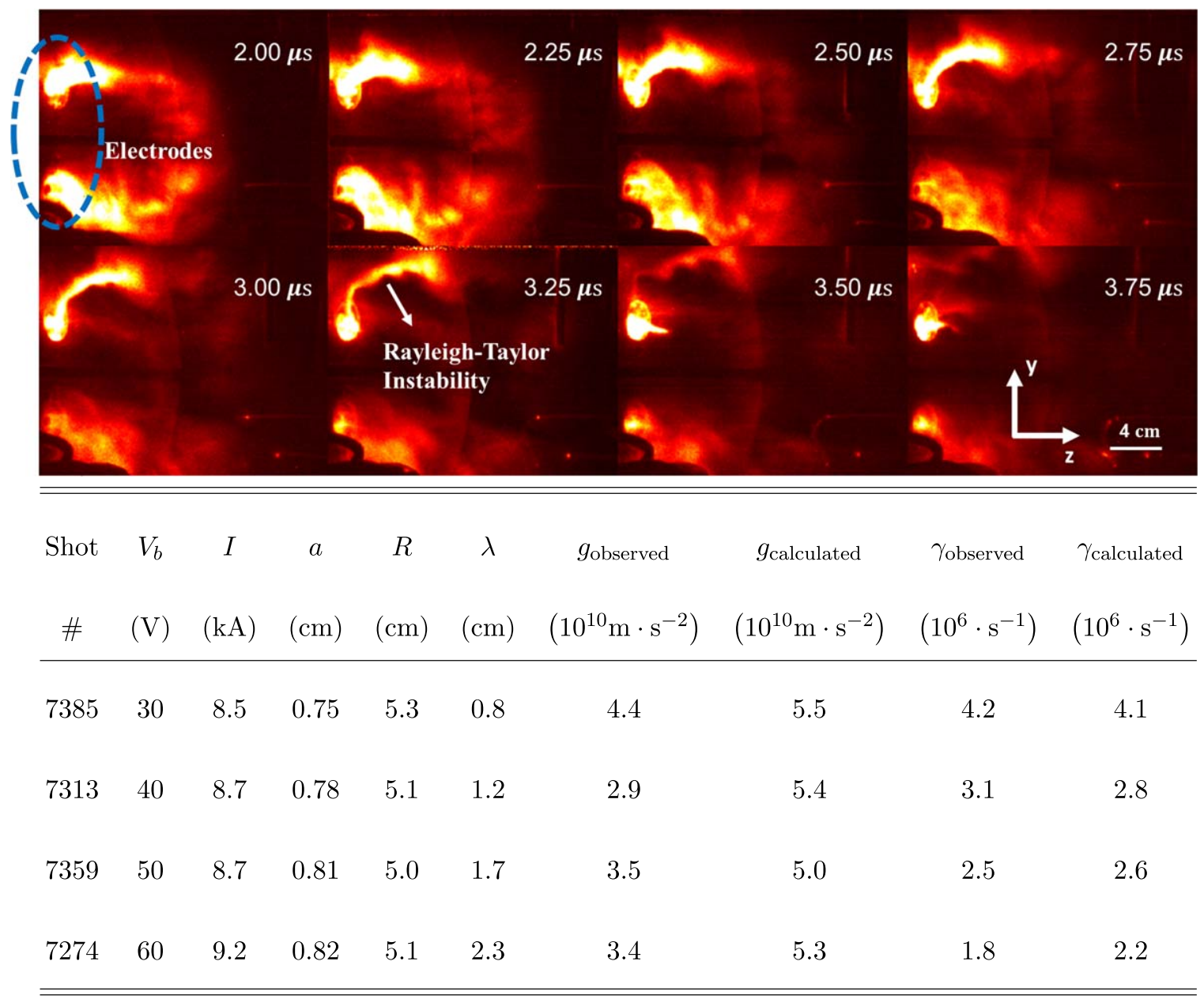

Figure 2. MRTI in time series images of $\mathrm{N}_{2}$ plasma loop evolution (these images are rotated $90^{\circ}$ clockwise compared with Figure 1). The table shows comparison between the observed growth rate and the calculated growth rate from the MRTI theory and comparison between the observed acceleration and the calculated acceleration from hoop force theory.

which gives an acceleration

$$
g_{\text {hoop }}=\frac{f_{\text {hoop }}}{\rho \pi a^{2}}=\frac{\mu_{0} I^{2}}{4 \pi^{2} \rho a^{2} R}\left[\ln \left(\frac{R}{a}\right)+1.33\right] .
$$

$R$ and $a$ are obtained from the images and $I$ is obtained from a Rogowski coil measurement. This provides sufficient information to compare the observed acceleration $g_{\text {observed }}$ with the calculated acceleration $g_{\text {calculated }}$ from the hoop force. The MRTI occurs at $t=2-3 \mu \mathrm{s}$ and parameters measured at $t=2 \mu \mathrm{s}$ are used with density assumed to be $n=2 \times 10^{21} \mathrm{~m}^{-3}$ (Ha \& Bellan 2016; Haw 2018). The gas used is $\mathrm{N}_{2}$. The table in Figure 2 shows that the observed acceleration and the calculated acceleration have order of magnitude agreement. Discrepancies exist presumably because the current loop in the experiment is not a complete circle. Also, the measured current does not fully flow through the visible loop (Stenson 2012; Ha \& Bellan 2016); this is consistent with the observed acceleration being smaller than the calculated one.

The bias voltage $V_{b}$ (applied on the solenoids) controls the background axial magnetic field of the loop. When we increase the bias voltage so as to increase the axial magnetic field, we find the wavelength of the MRTI increases, as shown in Figure 3(a). For a sufficiently large bias magnetic field, the MRTI is not observed.
Figure 3(b) shows measurements for a sequence of bias fields $V_{b}=30,40,50,60 \mathrm{~V}$, each repeated for 10 shots. This plot indicates that for increasing bias voltage (which is proportional to the axial magnetic field), the wavelength increases.

This phenomenon is consistent with the MRTI theory, since Equation (5) shows that the increase of magnetic field causes the corresponding fastest-growing wavelength to increase. For a sufficiently large magnetic field, the wavelength is larger than the loop dimensions and the MRTI cannot occur. This is most likely the reason for the absence of the MRTI in previous solar loop simulation experiments where a much larger axial magnetic field was used (e.g., Stenson \& Bellan 2012; Wongwaitayakornkul et al. 2017). In Equation (5), besides $B_{0}$, the other two parameters, $\rho, g$, also influence the fastestgrowing wavelength. In order to remove the influence of these parameters and verify that the increasing wavelength corresponds with the MRTI theory, we introduce the parameter $s=\lambda \rho g$. Using Equation (5), the fastest-growing mode corresponds to

$$
s=\frac{8 \pi}{\mu_{0}} B_{0}^{2}=\frac{8 \pi \alpha^{2}}{\mu_{0}} V_{b}^{2} .
$$

To determine $s$, the quantities $\lambda$ and $g$ are obtained from the image measurement, while $n$ is determined as a relative density 

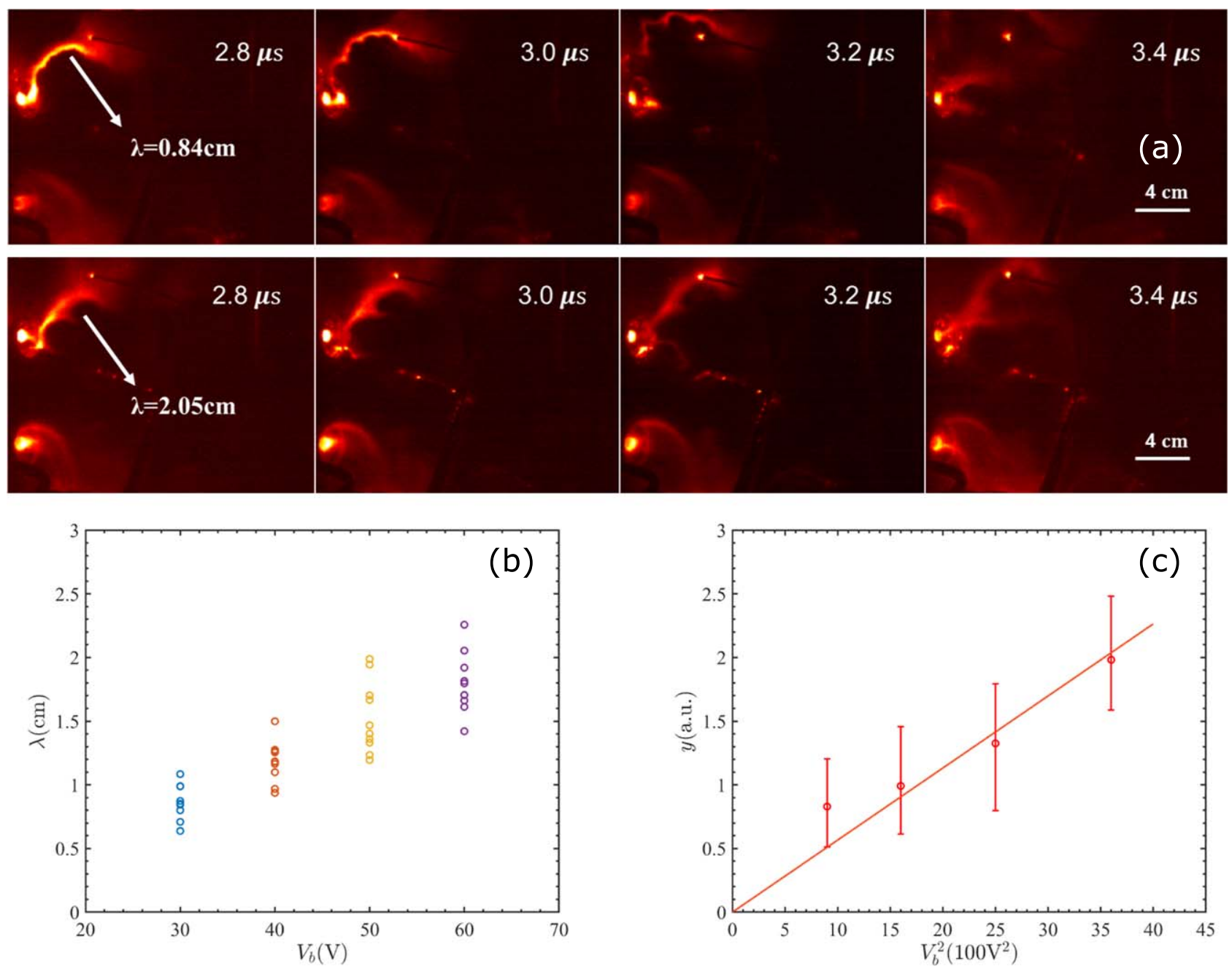

Figure 3. (a) Time series images of $\mathrm{N}_{2}$ plasma loop evolution (upper shot $\# 7385$ and $V_{b}=30 \mathrm{~V}$, lower shot $\# 7281$ and $V_{b}=60 \mathrm{~V}$ ). (b) Plot of the wavelength of the MRTI vs. different bias voltage. (c) Plot of $s$ vs. $\mathrm{V}_{b}^{2}$ (each data point is from 10 shots and the error bar represents the spread over these shots).

from the light intensity that scales as $n^{2}$. Keeping other experimental parameters the same, we set $V_{b}=30,40,50,60 \mathrm{~V}$ for 10 shots at each voltage to obtain $s$. Figure 3(c) shows $s$ plotted versus $V_{b}^{2}$. The error bars are determined by the shot-toshot variation of measured data and the line shows the best fit. From this figure, it is clear that $s$ scales as $V_{b}^{2}$ as predicted by Equation (10).

As the geometry of the experiment is more like a cylinder, the experimental results can also be used to compare with the MRTI under cylindrical geometry derived by Zhai \& Bellan (2016). Using the notation in Zhai \& Bellan (2016), the growth rate is

$$
\gamma=\gamma\left(x, \alpha, \Phi^{2}\right)
$$

where $x=k a=2 \pi a / \lambda, \alpha=B_{z} / B_{\theta}, \Phi^{2}=\mu_{0} \rho g a / B_{\theta}^{2}$, and $a$ is the radius of the cylinder. Consider a system with certain values $\Phi^{2}$ and $\alpha$ subject to a random perturbation that contains all possible $x=k a$. The component $x$ that gives the largest $\gamma=\gamma\left(x, \alpha, \Phi^{2}\right)$ is defined as $x^{*}$, and the fastest growth rate is defined as $\gamma^{*}$. Figure 4(a) shows the dependence of the fastest-growing mode on $\alpha$ for fixed $\Phi^{2}=3.77$. Note that $\alpha$ is proportional to the axial magnetic field and $x$ corresponds to $1 / \lambda$. Figure 4(a) shows that with the increase of axial magnetic field, the wavelength increases and the growth rate decreases, the same trend as predicted for the MRTI in planar geometry.

We assumed all of the experiments have the same parameters except for axial field with $\rho=5 \times 10^{-5} \mathrm{~m}^{-3}, g=3 \times$ $10^{10} \mathrm{~m} \mathrm{~s}^{-2}, I=10^{4} \mathrm{~A}, B_{\theta}=\mu_{0} I / 2 \pi a$, and that the observed mode is the fastest-growing mode. The only free parameter in the determination of $\Phi^{2}$ is $a$. From the experimental results, $x^{*}$ can be obtained and the corresponding $\alpha$ and $\gamma^{*}$ can be calculated. The observed growth rate and the calculated growth rate are compared in Figures 4(b)-(f) for different $a$. For $a=0.015-0.025 \mathrm{~m}$, the observed growth rate agrees well with the MRTI theory in cylindrical geometry. The value for the radius is somewhat larger than the observed $0.008 \mathrm{~m}$ value. This discrepancy may result from simplifying theoretical assumptions such as uniform density and axial magnetic field and current flowing through the surface. In Zhai \& Bellan (2016), the radius used for comparison was also larger than the one observed from the image. 

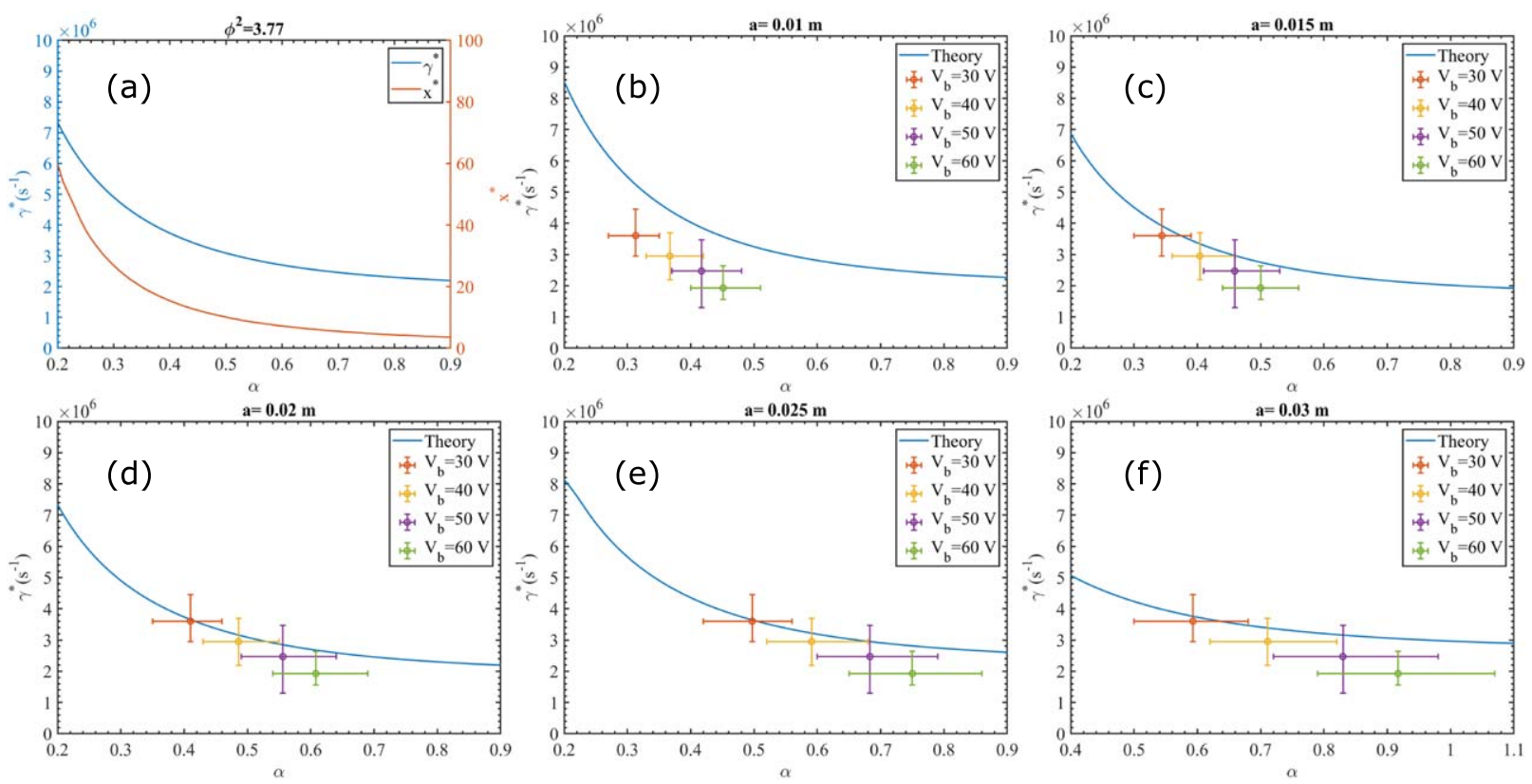

Figure 4. (a) $x^{*}$ and $\gamma^{*}$ vs. $\alpha$ with $\Phi^{2}=3.77$. (b)-(f) Comparison between the observed growth rate and the calculated growth rate from the MRTI theory under cylindrical geometry (each data point is from 10 shots and the error bar represents the spread over these shots).
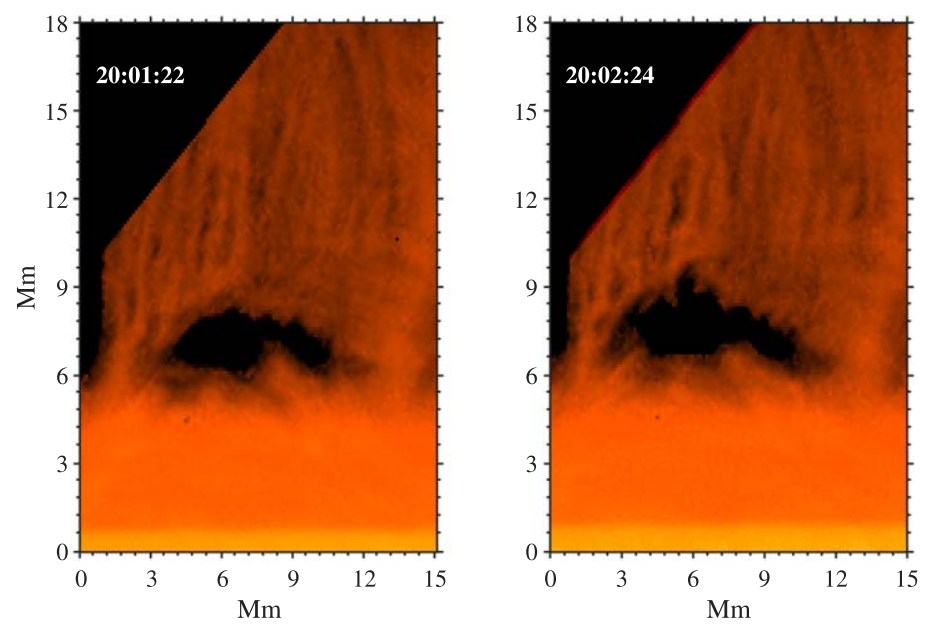

\begin{tabular}{cccc}
\hline \hline & Experiment & Solar quiescent & Scaled \\
& & prominence & experiment \\
\hline$L(\mathrm{~m})$ & $10^{-2}$ & $10^{6}$ & $10^{6}$ \\
$t(\mathrm{~s})$ & $10^{-6}$ & 60 & 78 \\
$B(\mathrm{~T})$ & 0.3 & $1.2 \times 10^{-3}$ & $1.2 \times 10^{-3}$ \\
$\rho\left(\mathrm{kg} \cdot \mathrm{m}^{-3}\right)$ & $5 \times 10^{-5}$ & $5 \times 10^{-10}$ & $5 \times 10^{-10}$ \\
$P(\mathrm{~Pa})$ & $3 \times 10^{3}$ & $5 \times 10^{-2}$ & $5 \times 10^{-2}$ \\
$v_{A}\left(\mathrm{~m} \cdot \mathrm{s}^{-1}\right)$ & $3.8 \times 10^{4}$ & $4.8 \times 10^{4}$ & $4.9 \times 10^{4}$ \\
$\beta$ & 0.08 & 0.09 & 0.08 \\
$g\left(\mathrm{~m} \cdot \mathrm{s}^{-2}\right)$ & $3 \times 10^{10}$ & 274 & 500 \\
\hline \hline
\end{tabular}

Figure 5. MRTI in a quiescent prominence observed on 2007 August 8 at 20:01:22 and 20:02:24 UT in the H $\alpha$ line from the Hinode Solar Optical Telescope (Kosugi et al. 2007). The table shows dimensionless scaling of the experiment to solar prominences. 


\section{Conclusions and Discussions}

In conclusion, we have determined a hoop force driven MRTI in a laboratory experiment. Changing the axial magnetic field shows that the wavelength of the MRTI increases with the increase of axial magnetic field. This scaling is verified to be consistent with both the planar MRTI theory and the more detailed cylindrical MRTI theory.

For the experiment, the characteristic length is $L=0.01 \mathrm{~m}$ and the characteristic time is $\tau=10^{-6} \mathrm{~s}$. Plasma density is about $2 \times 10^{21} \mathrm{~m}^{-3}$. Plasma temperature is assumed to be $5 \mathrm{eV}$. The corresponding Debye length $\lambda_{\mathrm{D}}=\sqrt{\frac{\varepsilon_{0} k_{\mathrm{B}} T}{n e^{2}}}=3.7 \times 10^{-7} \mathrm{~m} \ll L$ and $4 \pi n \lambda_{\mathrm{D}}^{2} / 3=430 \gg 1$. The resistive diffusion time is $\mu_{0} L^{2} / \eta=2 \times 10^{-6} \mathrm{~s}>\tau$, where the Spitzer resistivity $\eta=$ $6.8 \times 10^{-5} \Omega \mathrm{m}$. There may be some magnetic field diffusion effects. Near the MRTI region, the axial magnetic field is measured to be $0.04-0.1 \mathrm{~T}$ when there is no plasma. The toroidal magnetic field generated by the current is calculated to be $0.2-0.4$ $\mathrm{T}$, so we assume a nominal total magnetic field $\sim 0.3 \mathrm{~T}$. For $B=0.3 \mathrm{~T}$, the cyclotron frequency for nitrogen is then $\omega_{\mathrm{ci}}=2 \times 10^{6} \mathrm{~s}^{-1}$ while the characteristic time is $\tau=10^{-6} \mathrm{~s}$. The system is thus marginally in the MHD regime. Hall term corrections may thus alter the MRTI dispersion relation derived from ideal MHD. We do not insert magnetic probes to measure the magnetic field at the MRTI location because the MRTI scale is so small that a probe will disturb the MRTI. As the MRTI dispersion relation derived from ideal MHD can describe this phenomenon properly, we assume the ideal MHD provides a reasonable description to the lowest order.

The MRTI behavior reported here is likely to apply to many other situations governed by MHD because MHD has no intrinsic length scale. MHD scaling (Ryutov et al. 2000) allows for three free parameters: $a_{1}, a_{2}, a_{3}$, following invariant relations: $\frac{L_{0}}{a_{1}} \rightarrow L^{\prime}, \frac{\rho_{0}}{a_{2}} \rightarrow \rho^{\prime}, \frac{B_{0}}{\sqrt{a_{3}}} \rightarrow B^{\prime}, \frac{P_{0}}{a_{3}} \rightarrow P^{\prime}, \frac{1}{a_{1}} \sqrt{\frac{a_{3}}{a_{2}}} t \rightarrow t^{\prime}$, $\sqrt{\frac{a_{2}}{a_{3}}} v_{0} \rightarrow v^{\prime}, \frac{a_{1} a_{2}}{a_{3}} g_{0} \rightarrow g^{\prime}$ to transform a scale to another scale having the same plasma beta. This transformation gives a oneto-one correspondence between systems, allowing laboratory experimental plasmas to be scaled to equivalent systems in space plasmas.

Figure 5 shows the MRTI in a quiescent prominence observed on 2007 August 8 UT in the $\mathrm{H} \alpha$ line from the Hinode Solar Optical Telescope (Kosugi et al. 2007). The mean value of initial wavelength is about $1 \mathrm{Mm}$ and the characteristic time of the MRTI is about 1 minute (Berger et al. 2010). For a typical solar prominence, a representative value for mass density is $5 \times 10^{-10} \mathrm{~kg} \mathrm{~m}^{-3}$ (Hirayama 1986). The pressure is in the range $0.01-0.1 \mathrm{~Pa}$ and a representative value is $0.05 \mathrm{~Pa}$ (Hanssen 1995). The magnetic field is about 3-26 G and a representative value is $12 \mathrm{G}$ (Leroy 1988).

The table in Figure 5 shows characteristic parameters of the experiment, the solar quiescent prominence, and the experiment scaled to the solar prominence using $a_{1}=10^{-8}$, $a_{2}=1 \times 10^{5}, a_{3}=6 \times 10^{4}$. The scaled characteristic parameters have magnitudes similar to that of a solar quiescent prominence. From these results, the variation of magnetic field strength may be a reason for observed differences in plume dynamics in solar prominences, so that prominences having weak magnetic field produce many small plumes while those with strong magnetic fields produce large plumes.

This material is based upon work supported by the NSF Solar Terrestrial Research Program via award grant 1914599 and by the Air Force Office of Scientific Research Space Science Program via award grant FA9550-17-1-0023. Hinode is a Japanese mission developed and launched by ISAS/JAXA, with NAOJ as domestic partner and NASA and STFC (UK) as international partners. It is operated by these agencies in cooperation with ESA and NSC (Norway).

\section{ORCID iDs}

Yang Zhang iㅏ https://orcid.org/0000-0002-4168-9225 Pakorn Wongwaitayakornkul (1) https://orcid.org/0000-00017455-8582

Paul M. Bellan (10 https://orcid.org/0000-0002-0886-8782

\section{References}

Berger, T. E., Slater, G., Hurlburt, N., et al. 2010, ApJ, 716, 1288 Betti, R., Goncharov, V., McCrory, R., \& Verdon, C. 1998, PhPl, 5, 1446 de Grouchy, P., Kusse, B., Banasek, J., et al. 2018, PhPl, 25, 072701 Goedbloed, H., Keppens, R., \& Poedts, S. 2019, Magnetohydrodynamics: Of Laboratory and Astrophysical Plasmas (Cambridge: Cambridge Univ. Press)

Ha, B. N., \& Bellan, P. M. 2016, GeoRL, 43, 9390

Ha, Q. B. N. 2016, PhD thesis, California Institute of Technology

Hachisu, I., Matsuda, T., Nomoto, K., \& Shigeyama, T. 1992, ApJ, 390, 230

Hansen, J. F., \& Bellan, P. M. 2001, ApJL, 563, L183

Hanssen, E. T. 1995, The Nature of Solar Prominences (Dordrecht: Springer) Haw, M. A. 2018, PhD thesis, California Institute of Technology

Haw, M. A., Wongwaitayakornkul, P., Li, H., \& Bellan, P. M. 2018, ApJL, 862, L15

Hester, J. J., Stone, J. M., Scowen, P. A., et al. 1996, ApJ, 456, 225

Hillier, A. 2018, RvMPP, 2, 1

Hillier, A., Berger, T., Isobe, H., \& Shibata, K. 2012, ApJ, 746, 120

Hirayama, T. 1986, NASCP, 2442, 149

Keppens, R., Xia, C., \& Porth, O. 2015, ApJL, 806, L13

Kosugi, T., Matsuzaki, K., Sakao, T., et al. 2007, The Hinode Mission (Berlin: Springer), 5

Kruskal, M. D., \& Schwarzschild, M. 1954, RSPSA, 223, 348

Lebedev, S., Mitchell, I., Aliaga-Rossel, R., et al. 1998, PhRvL, 81, 4152

Leroy, J. 1988, Dynamics and Structure of Quiescent Solar Prominences (Berlin: Springer), 77

Porth, O., Komissarov, S. S., \& Keppens, R. 2014, MNRAS, 443, 547

Ryutov, D., Drake, R., \& Remington, B. 2000, ApJS, 127, 465

Ryutova, M., Berger, T., Frank, Z., et al. 2010, SoPh, 267, 75

Shafranov, V. 1966, RvPP, 2, 103

Sinars, D., Slutz, S., Herrmann, M., et al. 2010, PhRvL, 105, 185001

Stenson, E. V. 2012, PhD thesis, California Institute of Technology

Stenson, E. V., \& Bellan, P. M. 2012, PhRvL, 109, 075001

Takabe, H., Mima, K., Montierth, L., \& Morse, R. 1985, PhFl, 28, 3676

Terradas, J., Soler, R., Luna, M., Oliver, R., \& Ballester, J. 2015, ApJ, 799, 94

Wongwaitayakornkul, P., Haw, M. A., Li, H., Li, S., \& Bellan, P. M. 2017, ApJ, 848, 89

Zhai, X., \& Bellan, P. M. 2016, PhPl, 23, 032121 